\title{
Macrophage Polarization in Health and Disease
}

\author{
Luca Cassetta, ${ }^{1}$ Edana Cassol, ${ }^{2}$ and Guido Poli ${ }^{3}$ \\ ${ }^{1}$ Department of Developmental and Molecular Biology, Center for the Study of \\ Reproductive Biology and Women's Health, Albert Einstein College of Medicine, \\ Bronx, NY 10461, USA \\ ${ }^{2}$ Department of Cancer Immunology and AIDS, Dana-Farber Cancer Institute, \\ 44 Binney Street, Boston, MA 02115, USA \\ ${ }^{3}$ AIDS Immunopathogenesis Unit, Division of Immunology, Transplantation and \\ Infectious Diseases, School of Medicine, San Raffaele Scientific Institute and \\ Vita-Salute San Raffaele University, P2/P3 Laboratories, DIBIT-1, Via Olgettina No. \\ 58, 20132 Milano, Italy
}

Received 30 October 2011; Accepted 9 November 2011

Academic Editor: Marco Antonio Cassatella

Macrophages are terminally differentiated cells of the mononuclear phagocyte system that also encompasses dendritic cells, circulating blood monocytes, and committed myeloid progenitor cells in the bone marrow. Both macrophages and their monocytic precursors can change their functional state in response to microenvironmental cues exhibiting a marked heterogeneity. However, there are still uncertainties regarding distinct expression patterns of surface markers that clearly define macrophage subsets, particularly in the case of human macrophages. In addition to their tissue distribution, macrophages can be functionally polarized into M1 (proinflammatory) and M2 (alternatively activated) as well as regulatory cells in response to both exogenous infections and solid tumors as well as by systems biology approaches.

KEYWORDS: Macrophage, polarization, M1/M2, HIV, tumors, TAMs (tumor-associated macrophages), regulatory macrophages 


\section{MACROPHAGE POLARIZATION: DOGMA OR REALITY?}

Proinflammatory, "classical Activation" of macrophages, which was delineated in early studies from the 1960s [1, 2], depends on the secreted molecules of activated T helper 1 (Th1) CD4 ${ }^{+}$lymphocytes or natural killer (NK) cells, and in particular of interferon- $\gamma(\mathrm{IFN}-\gamma)$, and of other proinflammatory agents such as tumor necrosis factor- $\alpha$ (TNF- $\alpha$ ) and bacterial lipopolysaccharide (LPS). Classical activation results in a population of macrophages with enhanced microbicidal capacity and increased secretion of proinflammatory cytokines to further strengthen the cell-mediated adaptive immunity $[3,4]$. In contrast, interleukin-4 (IL-4) and IL-13, signature cytokines of the CD4 ${ }^{+} \mathrm{Th} 2$ response, namely, are the major inducers of the "alternative activation" of macrophages that play an important role not only in the immune response to parasites, but also in allergy, wound healing, and tissue remodeling $[5,6]$.

Indeed, "classically" and "alternatively" activated macrophages have been designated as "M1" and "M2" macrophages, respectively, by analogy to the Th1/Th2 division of labor of CD4 helper T cells [7]. M2-polarized macrophages are further subdivided into M2a (elicited by IL-4 or IL-13), M2b (following stimulation by immune complexes in the presence of a Toll-like receptor ligand), and M2c (when exposed to anti-inflammatory stimuli such as glucocorticoid hormones, IL-10, or Transforming Growth Factor- $\beta$, TGF- $\beta$ ) [8]. Although this classification of macrophages provides a useful working scheme, it unlikely fully represents the complexity of the transitional states of macrophage activation, which is often fine tuned in response to different microenvironments.

A more flexible classification has been suggested recently by mouse studies in which macrophages are considered as part of a continuum having a range of overlapping functions and in which classically activated, wound-healing, and regulatory macrophages occupy different points of the spectrum [9]. However, also this classification does not take into account the role of macrophages during development encompassing embryonic [10] and wound-healing [9] macrophages as well as irreversibly differentiated osteoclasts [11]. The differentiation of these different macrophages is profoundly influenced by the microenvironment, although there is considerable plasticity between distinct cell types. This concept recalls Metchnikoff's original classification that considered macrophages as part of a continuum, keeping the self whole in development and adulthood (physiological inflammation) and differentiating it from nonself and the environment (pathological inflammation).

\section{MACROPHAGE PLASTICITY: AN OBSTACLE TO STUDY MACROPHAGE POLARIZATION?}

Unlike lymphocytes where phenotypic changes are largely "fixed" by chromatin modifications after exposure to polarizing cytokines, macrophages have a plastic gene expression profile that is influenced by the type, concentration, and longevity of exposure to the stimulating agents, as documented extensively $[9,12-14]$. For example, M2 macrophages can be readily induced to express M1-associated genes by exposure to TLR ligands or IFN- $\gamma$ [15-17]. Indeed, gene expression plasticity was the rule rather than the exception when experimental polarization was performed [15-17]. In this regard, gene expression plasticity represents an adaptive response to different microenvironmental stimuli, in which macrophages migrate in response to chemotactic signals or chemokines, phagocytose dead cells and debris, and functionally interact with different $\mathrm{T}$ cell subsets.

Macrophages are renown for their apparent phenotypic heterogeneity and for the diverse activities in which they engage $[18,19]$. Many of these activities appear to be opposing in nature: pro- versus antiinflammatory effects, immunogenic versus tolerogenic activities, and tissuedestruction versus tissue-repair $[6,18,19]$. Indeed, significant differences between genes expressed early (up to $6 \mathrm{~h}$ ) versus late (12-24 h or later) after LPS stimulation have been reported [20]. Thus, the functional pattern expressed by macrophages changes with time as the response progresses. It should also be noted that macrophages frequently respond to the early cytokines that they secrete in an autocrine/paracrine fashion. Macrophages treated overnight with IL-4 prior to stimulation with LPS display enhanced production of TNF- $\alpha$ and IL-12, in stark contrast 
to the reduced production of these cytokines observed upon stimulation with LPS in the presence of IL-4 [21].

There is a high number of factors contributing to diversity of macrophage function, including the synergistic or antagonistic effects of different cytokines and related signals on their differential expression, chemokines, hormones (including adrenergic and cholinergic agonists), TLR ligands, and other endogenous ligands (e.g., histamine, integrin ligands, peroxisome proliferator-activated receptor ligands, apoptotic cells); this plethora of signals underlines the fact that macrophages can display a large number of distinct, functional patterns that have not yet been completely defined. Furthermore, identical macrophages placed in different microenvironments display different functions in response to a common stimulus. Stimulation of macrophages with functionally opposite cytokines, such as IFN- $\gamma$ and IL-4, initiates signal cascades that results in differential modulation (enhancement or inhibition) of different genes at the transcriptional or posttranscriptional level (e.g., stabilization or destabilization of mRNA). Unless the signal cascade triggered an apoptotic cascade, macrophages will eventually revert to their original, functional status after the cytokine signaling ceases.

In vivo or in vitro treatment of macrophages with cytokines alters their functional response pattern to LPS. However, if the cytokines are washed away after incubation and macrophages are then maintained in the absence of cytokines for 1-2 days before LPS stimulation, the functional response pattern is usually identical to that of macrophages that had not been prestimulated with the cytokine. A similar reversion to basal macrophage phenotype is observed when IL-4 and granulocyte macrophage-colony-stimulating factor (GM-CSF) are removed from human monocyte-derived, immature dendritic cells (iDCs) and the cells are resuspended in a neutral environment [22].

Therefore, most Th1 and Th2 cytokines do not seem to induce a stable differentiation of macrophages into distinct subsets, but they rather promote a transient functional pattern of responses that return to basal levels in a few (3-7) days.

\section{MARKERS OF MACROPHAGE POLARIZATION: STILL AN OPEN CHASE}

One of the most debated issues in the context of human macrophage polarization is the identification of unique or restricted markers to be used for research and clinical purposes. Innovative approaches, including intravital imaging and other in vivo techniques, will be of great help in the identification of "real" subsets of macrophages in addition to more static antigens expressed on their cellular surface following cell polarization. An example of this broader approach is summarized by the identification of at least 6 different subsets of mouse tumor-associated macrophages (TAMs) based on their distinct functional features (Table 1), as reviewed in [24]. According to this view, every macrophage subset not only expresses different cytokines and cytokine receptors but also plays a complete distinct role in tumor pathogenesis and evolution, from tumor initiation to tumor metastasis.

\section{CONTRIBUTION OF MACROPHAGE POLARIZATION TO INNATE IMMUNE RESPONSES}

Mononuclear phagocytes play a pivotal role in the establishment of antiviral immune responses. Unlike mDCs that capture antigens in mucosal tissues and then migrate to distal lymph nodes to activate specific T-cell responses, tissue macrophages are residential cells. Macrophages constitute up to $20 \%$ of all mononuclear cells present in mucosal tissues and play an important role in activating localized responses [43].

While M1 and M2 macrophages facilitate the development of Th1 and Th2 responses, respectively $[3,9,19,44]$, their role in the modulation of natural killer (NK) and T regulatory (Treg) immunosuppressive remains undefined. NK cells are a critical component of innate immune response. They promote antiviral immunity by killing infected cells, via production of inflammatory cytokines and by interactions with $\mathrm{T}$ cells with DC, a process that shapes the magnitude and quality of adaptive immune responses [45]. A recent 
TABLE 1: Subpopulations of macrophages in different pathologies.

\begin{tabular}{|c|c|c|}
\hline Disease & Markers & References \\
\hline \multicolumn{3}{|l|}{ Cancer } \\
\hline Invasive macrophages & $\mathrm{WNT}^{+}, \mathrm{EGF}^{+}$ & [25-28] \\
\hline Activated macrophages & $\mathrm{IL}_{-1} 2^{+}, \mathrm{MHC} \mathrm{II}^{\mathrm{HI}}, \mathrm{TNF}-\alpha^{+}, \mathrm{CD} 80 / 86^{+}$ & {$[29,30]$} \\
\hline Immunosuppressive macrophages & Arginase $^{+}, \mathrm{MARCO}^{+}, \mathrm{IL}^{-10^{+}}, \mathrm{CCL}-22^{+}$ & {$[31,32]$} \\
\hline Angiogenic macrophages & $\begin{array}{l}\text { VEGFR } 1^{+}, \mathrm{VEGF}^{+}, \mathrm{CXCR}^{+}, \mathrm{Tie}^{+}{ }^{+}, \\
\mathrm{EST}^{+}{ }^{+}\end{array}$ & [33-36] \\
\hline Metastasis-associated macrophages & $\mathrm{VEGFR}^{+}, \mathrm{CXCR}^{-}, \mathrm{CCR} 2^{+}, \mathrm{Tie} 2^{-}$ & {$[24,37-39]$} \\
\hline $\begin{array}{l}\text { FoxP } 3^{+} \text {macrophages (regulatory } \\
\text { macrophages) }\end{array}$ & $\begin{array}{l}\text { PGE }_{2}, \text { Arg-2, IL-1 } \alpha, \text { CXCL4, CCL7, } \\
\text { CCL9, CXCL12, CXCL13, PDGF, and } \\
\text { VEGF }\end{array}$ & {$[40]$} \\
\hline \multicolumn{3}{|l|}{$H I V$} \\
\hline HIV-transmitting macrophages & $\begin{array}{l}\text { DC-SIGN }{ }^{+}, \text {CD163, CD206, High } \\
\text { Phagocytosis }\end{array}$ & (Cassol et al., submitted) \\
\hline HIV-resistant macrophages & APOBEC $3 \mathrm{~A}^{+}$, Low phagocytosis & (Cassetta et al., submitted) \\
\hline \multicolumn{3}{|l|}{ Other } \\
\hline Perivascular macrophages & Phagocytosis & {$[41,42]$} \\
\hline
\end{tabular}

study by Romo et al. showed that both M1 and M2 cells were able to trigger NK cell degranulation but that only M1 cells infected with the human cytomegalovirus efficiently promoted NK-cell-mediated IFN- $\gamma$ production [46]. Further studies will be required to understand if similar events occur in the case of other viral infections.

If Treg cells, on the one hand, are potent suppressors of the adaptive immune response, on the other hand it is less know how they affect the innate immune system. Tiemessen et al. showed that Tregs can steer monocyte differentiation towards alternative activation resulting in increased expression of CD163 and CD206, increased production of CCL18, and enhanced phagocytic capacity [47]. Interestingly, Savage et al. demonstrated that M2, but not M1, macrophages induced differentiation of Treg cells with a strong suppressive phenotype [48]. Their mechanism of action required cell-cell contact and involved membranebound TGF- $\beta 1$ expression [48]. Thus, macrophage polarization can indeed modulate multiple aspects of both the adaptive and innate immune responses.

\section{REGULATORY MACROPHAGES (RMs)}

As mentioned, diversity is a key feature of macrophage activation. In addition to M1 and M2 macrophages, RMs have recently emerged as an important population of cells that play a pivotal role in limiting inflammation during innate and adaptive immune responses [9, 44]. Similar to M2 macrophages, RMs produce high levels of IL-10; however, unlike M2 cells, they do not contribute to the production of extracellular matrix and express high levels of the costimulatory molecules CD80/B7-1 and CD86/B7-2 $[9,49]$. Studies in mice have demonstrated a crucial role for both IL-4/IL-13 and IFN- $\gamma$ signaling pathways in the induction of RM [50,51]. Furthermore, they have shown that regulatory cells concurrently express nitric oxide synthase and arginase (Arg) suggesting that they have a distinct activation phenotype [3]. Given the potent T-cell suppressive function of RM, it will be important to develop reliable biomarkers for their identification.

Interestingly, a recent study has identified a subpopulation of Foxp $3^{+}$macrophages in the mouse [40]. These cells, which are distributed throughout the major lymphoid organs, inhibit T-cell proliferation. 
Manrique et al. reported that $\mathrm{F} 4 / 80^{+} \mathrm{Foxp}^{-}$cells could be converted into FoxP3 ${ }^{+}$cells by stimulation with TGF- $\beta$, Vascular Endothelial Growth Factor (VEGF), or TLR ligands [40]. According to their study, Foxp $3^{+}$macrophages inhibit immune responses via both the secretion of soluble factors (such as prostaglandin $\mathrm{E}_{2}, \mathrm{PGE}_{2}$, arginase-2, Arg-2, IL-1 $\alpha$, CXCL4, CCL7, CCL9, CXCL12, CXCL13, platelet derived growth factor, and VEGF) and induction of cell death by increased expression of TRAIL, CD200r, LAG3, B7-H1, B7H-4, and PD1 [40]. Foxp3 ${ }^{+}$macrophages also promote the development of Treg cells [40]. Based on cytokine and transcriptional profiles, the authors define these cells as "Foxp3 RM"; it is currently unclear if a similar population exists in humans.

Although cell activation is critical for the induction of an effective immune response to pathogens or tumors, inappropriate and sustained activation/polarization of macrophages leads to tissue damage, immune dysfunction, and disease. As with exacerbated M1 and M2 responses, dysfunctional regulatory responses contribute to tumor progression and growth (as discussed below) and can predispose the host to infection. Several pathogens including Staphylococcus aureus [52], Leishmania [53], and African Trypanosomas [54] exploit regulatory responses to facilitate immune escape and enhance their own survival in the host. For example, Leishmania binds and triggers $\mathrm{Fc} \gamma \mathrm{R}$ signaling during entry, resulting in the development of RM which are permissive to its intracellular growth [53]. Given their capacity to suppress adaptive immune responses, it will be important to understand more clearly how RMs contribute to dysfunctional immune responses in chronic viral infections such as HIV.

\section{MACROPHAGE DIVERSITY IN SYSTEMS BIOLOGY}

Systems biology approaches have provided important insights into the heterogeneity of mononuclear phagocyte populations, the plasticity of macrophage activation, and the molecular pathways associated with polarization. Transcriptome profiling has been commonly used to examine networks of molecules and transcription factors linked to activation. Using this approach, Martinez et al. obtained a comprehensive global view of human macrophage polarization [19]. Expanding on previous studies of M1 and M2 markers, they found that while M1 polarization was associated with dramatic changes in the transcriptome, M2 polarization resulted in minimal alterations in gene expression. The modulation of genes involved in cellular metabolic activities was a prominent feature of polarization. These genes included a set of apolipoproteins (APOL1, APOL2, APOL3, and APOL6) that play a central role in cholesterol transport [19]. More recent transcriptome studies have focused on understanding the spectrum of polarization phenotypes associated with disease. Pena et al. found that the range of changes observed during LPS tolerance, including the upregulation of genes important in wound repair (MMP-9, MMP-7, VEGF, FGF-2, and f-MLPR ligand-1), were similar to changes observed during M2 polarization [55]. This led the authors to propose that LPS tolerance represents a distinct state of $\mathrm{M} 2$ polarization.

Epigenetic studies have begun to unravel how polarized macrophages acquire and maintain their activation phenotype. M2 genes in mice, including Chi313, Retnla, and Arg-1, were shown to be epigenetically regulated as a result of signal transducer and activator of transcription 6-(STAT6-) dependent induction of the H3K27 demethylase Jmjd3 [56]. Furthermore, Satoh et al. found that Jmjd3 was a gene essential for M2, but not M1, macrophage polarization in response to helminthic infections [57]. They also identified Irf4 as a Jmjd3 target gene, a key transcription factor that controls M2 polarization. More recently, Zhang et al. observed that genes with a potential for increased/decreased expression after macrophage polarization (i.e., IFN- $\gamma$, IFN- $\alpha$, and IL-4) were generally enriched for cytokine-induced $\mathrm{H} 4$ acetylation (H4ac), a marker of increased transcriptional competence [58]. Furthermore, monocyte (and potentially macrophage) polarization was heterogenous with respect to durability. This systems approach identified mitogen-activated protein (MAP) kinases as central to the polarization process consistent recent studies highlighting the role of MAP kinases in histone modification [58].

Thus, systems biology will keep providing a constantly updated global view of the networks regulating or involved in macrophage polarization, allowing us to evaluate key issues related to macrophage heterogeneity and plasticity. 


\section{MACROPHAGE POLARIZATION IN CANCER BIOLOGY: A MATTER OF GOOD OR BAD EDUCATION}

All solid tumors recruit monocytes and local macrophages into their microenvironment making them TAM; it is being increasingly clear that TAMs play several, sometimes opposite, roles during tumor development. Originally it was believed that these cells were attempting to reject the immunologically non-self entity made of transformed cells (that frequently lose or modify their MHC profile). Indeed, macrophages can effectively kill tumor cells in vitro [59]. However, clinical and experimental evidence indicates that, in most cases, TAMs promote rather than counteract tumor progression, including their metastatic phase [25, 60, 61]. For example, the density of TAM in human tumors correlates with poor prognosis in $>80 \%$ of the cases [62].

These observations suggest that the tumor environment causes recruited macrophages to carry out trophic functions for cancer cells by adopting an M2-related profile. In fact, macrophages that differentiate in the presence of growth factors such as M-CSF/Colony-Stimulating Factor-1 (CSF-1) [63] or in response to stimuli of the nuclear factor $-\kappa \mathrm{B}(\mathrm{NF}-\kappa \mathrm{B})$ activation pathway $[64,65]$ are nonimmunogenic and exert trophic effects on the tumor growth. In this regard, it is important to underscore that TAMs found in progressing tumors are different from macrophages mediating chronic inflammatory responses to pathogens or irritants that may be involved in cancer initiation by creating a mutagenic and cell-growth-promoting environment $[66,67]$. Indeed, TAM isolated from progressing tumors are functionally similar to those found in developing tissues [68], therefore supporting the hypothesis that tumors can "diseducate" macrophages to facilitate tumor progression and invasion (Table 1).

In conclusion, tumors can affect macrophages playing with their impressive plastic nature in order to modify the microenvironment and consequently alter the function and the strength of the cellular and innate immune response. These studies together provide useful models to investigate how cancer cells (and viruses, as discussed later on) crosstalk with macrophages; the potential application of this information will be the identification of soluble factors or inhibitors that will "reeducate" macrophages against pathogens and neoplastic lesions.

\section{MACROPHAGE POLARIZATION IN HIV INFECTION AND OTHER VIRAL DISEASES}

The role of macrophage polarization in viral infections is far from being well defined. In this regard, a good example of macrophage education by a pathogen is represented by HIV-1. While in advanced patients the most prominent dysfunction is defective migratory responses of circulating monocytes to classical chemoattractants $[69,70]$, later linked to a downregulation of chemotactic receptors for C5a and for the bacterial tripeptide f-MLP [71, 72], monocytes as well as lung alveolar macrophages isolated from $\mathrm{HIV}^{+}$ individuals have also shown reduced phagocytic activity $[73,74]$, decreased phagosome-lysosome fusion, and decreased intracellular killing of opportunistic pathogens [73, 75]. These functional defects, in turn, result in the inefficient control of opportunistic pathogens and further enhancement of cell activation and disease pathogenesis.

Chronic HIV-1-associated immune activation also leads to altered secretion of pro- and antiinflammatory cytokines and chemokines and, ultimately, to dysregulation of the host immune system and the killing of bystander $\mathrm{CD}_{4}^{+} \mathrm{T}$ cells. In addition, HIV-infected macrophages have been implicated in the elimination of effector $\mathrm{CD}^{+} \mathrm{T}$ cells through interactions between TNF bound to the surface of macrophages and TNFRII expressed on $\mathrm{CD}^{+} \mathrm{T}$ cells [76]. Of interest, Brown et al. found that in vitro incubation with HIV-1 failed to induce classical macrophage activation via TLR, although the virus primed macrophages to become hyperresponsive to TLR ligands, a phenotype designated by the authors as "M1 $\mathrm{HIV}$ " [77]. 


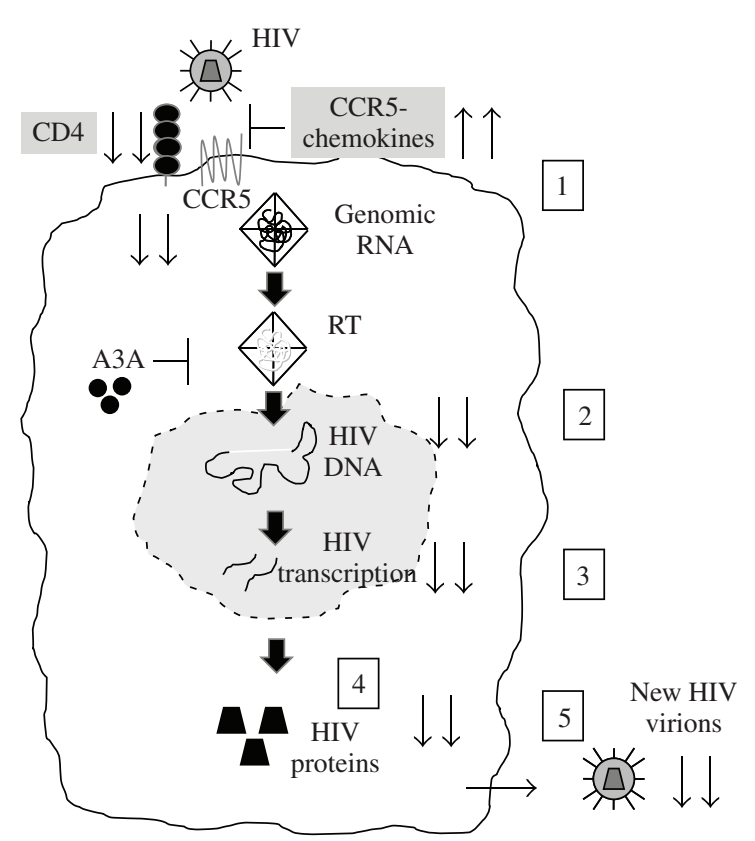

FIGURE 1: A model of M1-MDM as "HIV-1-resistant macrophages." (1) Downmodulation of CD4 and production of CCR5-binding chemokines that compete with gp120 Env for the ligation to the main viral entry coreceptor [23]. (2) Inhibition of viral DNA synthesis and proviral integration [23], (L. Cassetta et al. submitted). (3) Inhibition of proviral transcription (L. Cassetta et al., submitted). (4) Inhibition of HIV-1 protein synthesis [23], (L. Cassetta et al., submitted). (5) Inhibition of new particle assembly and release (hypothetical).

We have reported that MDM polarized in vitro towards M1 or M2a cells strongly inhibited HIV1 replication and production, although affecting different steps of the virus life cycle [23]. Based on our results, we can envisage M2a-polarized macrophages as "HIV-1-transmitting macrophages" in that characterized by the selective expression of the molecule known as dendritic cell-specific intercellular adhesion molecule-3-grabbing nonintegrin (DC-SIGN $\left.{ }^{+}, \mathrm{CD} 209\right)$ (that is otherwise downregulated in M1MDM, therefore representing a potentially useful marker for discriminating M1 and M2 macrophages). Indeed, although M2a show lower levels of virus replication than control MDM, they can transfer efficiently HIV-1 to CD4 ${ }^{+}$T cells via DC-SIGN (E. Cassol et al., submitted). In contrast, M1-MDM can be defined as "HIV-1-resistant macrophages" in that they show very low levels of virus replication as a result of both entry- and post-entry-dependent hurdles to the virus in order to complete its replicative cycle. In addition to being negative for DC-SIGN display at the cell surface, they are positive for the intracellular expression of apolipoprotein B mRNA editing enzyme, catalytic polypeptide-like 3A (APOBEC3A), a molecule that has been associated to an antiviral effect in monocytes [78] and, more recently, in MDM [79, 80] (L. Cassetta et al., submitted). The features typical of M1-MDM that are related to the restriction of HIV-1 replication are summarized in Figure 1.

\section{CONCLUSIONS}

The concept that, in addition to the adaptive response, also the complex network globally defined as "innate immuniaty" can be specialized and "pathogen specific," to some extent, led to the hypothesis that macrophage activation could follow different programs with the goal of combat distinct pathological situations or maintain a physiological homeostatic control of the local flora, as in the case of the gastrointestinal tract mucosa. Investigation of cancer, viral infections, and other pathological conditions will also inform on the plasticity and heterogeneity of macrophages and hopefully lead to better strategies to control and prevent the occurrence of these diseases. 


\section{AUTHORS' CONTRIBUTION}

L. Cassetta and E. Cassol equally contributed to this article.

\section{ACKNOWLEDGMENTS}

This study was supported by the following grants to G. Poli: CARIPLO Grant 2008-2230 and Grant no. $40 \mathrm{H} 76$ of the Program of AIDS research 2009-2010 of the Ministry of Health, Italy.

\section{REFERENCES}

[1] G. B. Mackaness, "The immunological basis of acquired cellular resistance," The Journal of Experimental Medicine, vol. 120, pp. 105-120, 1964.

[2] R. van Furth, Z. A. Cohn, J. G. Hirsch, J. H. Humphrey, W. G. Spector, and H. L. Langevoort, "The mononuclear phagocyte system: a new classification of macrophages, monocytes, and their precursor cells," Bulletin of the World Health Organization, vol. 46, no. 6, pp. 845-852, 1972.

[3] S. Gordon and P. R. Taylor, "Monocyte and macrophage heterogeneity," Nature Reviews Immunology, vol. 5, no. 12, pp. 953-964, 2005.

[4] S. Gordon, "The macrophage: past, present and future," European Journal of Immunology, vol. 37, no. 1, pp. S9-S17, 2007.

[5] S. Gordon, "Alternative activation of macrophages," Nature Reviews Immunology, vol. 3, no. 1, pp. 23-35, 2003.

[6] F. O. Martinez, L. Helming, and S. Gordon, "Alternative activation of macrophages: an immunologic functional perspective," Annual Review of Immunology, vol. 27, pp. 451-483, 2009.

[7] C. D. Mills, K. Kincaid, J. M. Alt, M. J. Heilman, and A. M. Hill, "M-1/M-2 macrophages and the Th1/Th2 paradigm," Journal of Immunology, vol. 164, no. 12, pp. 6166-6173, 2000.

[8] F. O. Martinez, A. Sica, A. Mantovani, and M. Locati, "Macrophage activation and polarization," Frontiers in Bioscience, vol. 13, no. 2, pp. 453-461, 2008.

[9] D. M. Mosser and J. P. Edwards, "Exploring the full spectrum of macrophage activation," Nature Reviews Immunology, vol. 8, no. 12, pp. 958-969, 2008.

[10] F. Rae, K. Woods, T. Sasmono et al., "Characterisation and trophic functions of murine embryonic macrophages based upon the use of a Csf1r-EGFP transgene reporter," Developmental Biology, vol. 308, no. 1, pp. 232-246, 2007.

[11] J. R. Edwards and G. R. Mundy, "Advances in osteoclast biology: old findings and new insights from mouse models," Nature Reviews Rheumatology, vol. 7, no. 4, pp. 235-243, 2011.

[12] S. K. Biswas, A. Sica, and C. E. Lewis, "Plasticity of macrophage function during tumor progression: regulation by distinct molecular mechanisms," Journal of Immunology, vol. 180, no. 4, pp. 2011-2017, 2008.

[13] S. Goerdt and C. E. Orfanos, "Other functions, other genes: alternative activation of antigen- presenting cells," Immunity, vol. 10, no. 2, pp. 137-142, 1999.

[14] R. D. Stout and J. Suttles, "Functional plasticity of macrophages: reversible adaptation to changing microenvironments," Journal of Leukocyte Biology, vol. 76, no. 3, pp. 509-513, 2004.

[15] R. D. Stout, C. Jiang, B. Matta, I. Tietzel, S. K. Watkins, and J. Suttles, "Macrophages sequentially change their functional phenotype in response to changes in microenvironmental influences," Journal of Immunology, vol. 175, no. 1, pp. 342-349, 2005.

[16] M. Modolell, I. M. Corraliza, F. Link, G. Soler, and K. Eichmann, "Reciprocal regulation of the nitric oxide synthase-arginase balance in mouse bone marrow-derived macrophages by TH1 and TH2 cytokines," European Journal of Immunology, vol. 25, no. 4, pp. 1101-1104, 1995.

[17] K. J. Mylonas, M. G. Nair, L. Prieto-Lafuente, D. Paape, and J. E. Allen, "Alternatively activated macrophages elicited by helminth infection can be reprogrammed to enable microbial killing," Journal of Immunology, vol. 182, no. 5, pp. 3084-3094, 2009. 
[18] D. A. Hume, "Macrophages as APC and the dendritic cell myth," Journal of Immunology, vol. 181, no. 9, pp. 5829-5835, 2008.

[19] F. O. Martinez, S. Gordon, M. Locati, and A. Mantovani, "Transcriptional profiling of the human monocyteto-macrophage differentiation and polarization: new molecules and patterns of gene expression," Journal of Immunology, vol. 177, no. 10, pp. 7303-7311, 2006.

[20] C. A. Wells, T. Ravasi, G. J. Faulkner et al., "Genetic control of the innate immune response," BMC Immunology, vol. 4, article 5, 2003.

[21] A. D'Andrea, X. Ma, M. Aste-Amezaga, C. Paganin, and G. Trinchieri, "Stimulatory and inhibitory effects of interleukin (IL)-4 and IL-13 on the production of cytokines by human peripheral blood mononuclear cells: priming for IL-12 and tumor necrosis factor $\alpha$ production," The Journal of Experimental Medicine, vol. 181, no. 2, pp. 537-546, 1995.

[22] G. Häusser, B. Ludewig, H. R. Gelderblom, Y. Tsunetsugu-Yokota, K. Akagawa, and A. Meyerhans, "Monocytederived dendritic cells represent a transient stage of differentiation in the myeloid lineage," Immunobiology, vol. 197, no. 5, pp. 534-542, 1997.

[23] E. Cassol, L. Cassetta, C. Rizzi, M. Alfano, and G. Poli, "M1 and M2a polarization of human monocyte-derived macrophages inhibits HIV-1 replication by distinct mechanisms," Journal of Immunology, vol. 182, no. 10, pp. 6237-6246, 2009.

[24] B. Z. Qian and J. W. Pollard, "Macrophage diversity enhances tumor progression and metastasis," Cell, vol. 141, no. 1, pp. 39-51, 2010.

[25] J. Condeelis and J. W. Pollard, "Macrophages: obligate partners for tumor cell migration, invasion, and metastasis," Cell, vol. 124, no. 2, pp. 263-266, 2006.

[26] J. Wyckoff, W. Wang, E. Y. Lin et al., "A paracrine loop between tumor cells and macrophages is required for tumor cell migration in mammary tumors," Cancer Research, vol. 64, no. 19, pp. 7022-7029, 2004.

[27] J. B. Wyckoff, Y. Wang, E. Y. Lin et al., "Direct visualization of macrophage-assisted tumor cell intravasation in mammary tumors," Cancer Research, vol. 67, no. 6, pp. 2649-2656, 2007.

[28] L. S. Ojalvo, C. A. Whittaker, J. S. Condeelis, and J. W. Pollard, "Gene expression analysis of macrophages that facilitate tumor invasion supports a role for Wnt-signaling in mediating their activity in primary mammary tumors," Journal of Immunology, vol. 184, no. 2, pp. 702-712, 2010.

[29] T. Enzler, S. Gillessen, J. P. Manis et al., "Deficiencies of GM-CSF and interferon $\gamma$ link inflammation and cancer," The Journal of Experimental Medicine, vol. 197, no. 9, pp. 1213-1219, 2003.

[30] L. Deng, J. F. Zhou, R. S. Sellers et al., "A novel mouse model of inflammatory bowel disease links mammalian target of rapamycin-dependent hyperproliferation of colonic epithelium to inflammation-associated tumorigenesis," American Journal of Pathology, vol. 176, no. 2, pp. 952-967, 2010.

[31] D. I. Gabrilovich and S. Nagaraj, "Myeloid-derived suppressor cells as regulators of the immune system," Nature Reviews Immunology, vol. 9, no. 3, pp. 162-174, 2009.

[32] F. Pucci, M. A. Venneri, D. Biziato et al., "A distinguishing gene signature shared by tumor-infiltrating Tie2expressing monocytes, blood "resident" monocytes, and embryonic macrophages suggests common functions and developmental relationships," Blood, vol. 114, no. 4, pp. 901-914, 2009.

[33] Y. N. Kimura, K. Watari, A. Fotovati et al., "Inflammatory stimuli from macrophages and cancer cells synergistically promote tumor growth and angiogenesis," Cancer Science, vol. 98, no. 12, pp. 2009-2018, 2007.

[34] S. Gazzaniga, A. I. Bravo, A. Guglielmotti et al., "Targeting tumor-associated macrophages and inhibition of MCP-1 reduce angiogenesis and tumor growth in a human melanoma xenograft," Journal of Investigative Dermatology, vol. 127, no. 8, pp. 2031-2041, 2007.

[35] E. Y. Lin, J. F. Li, G. Bricard et al., "Vascular endothelial growth factor restores delayed tumor progression in tumors depleted of macrophages," Molecular Oncology, vol. 1, no. 3, pp. 288-302, 2007.

[36] E. Y. Lin, J. F. Li, L. Gnatovskiy et al., "Macrophages regulate the angiogenic switch in a mouse model of breast cancer," Cancer Research, vol. 66, no. 23, pp. 11238-11246, 2006.

[37] R. N. Kaplan, R. D. Riba, S. Zacharoulis et al., "VEGFR1-positive haematopoietic bone marrow progenitors initiate the pre-metastatic niche," Nature, vol. 438, no. 7069, pp. 820-827, 2005. 
[38] S. Hiratsuka, A. Watanabe, Y. Sakurai et al., "The S100A8-serum amyloid A3-TLR4 paracrine cascade establishes a pre-metastatic phase," Nature Cell Biology, vol. 10, no. 11, pp. 1349-1355, 2008.

[39] J. A. Joyce and J. W. Pollard, "Microenvironmental regulation of metastasis," Nature Reviews Cancer, vol. 9, no. 4, pp. 239-252, 2009.

[40] S. Z. Manrique, M. A. D. Correa, D. B. Hoelzinger et al., "Foxp3-positive macrophages display immunosuppressive properties and promote tumor growth," The Journal of Experimental Medicine, vol. 208, no. 7, pp. 1485-1499, 2011.

[41] B. Gligorijevic, D. Kedrin, J. E. Segall, J. Condeelis, and J. van Rheenen, "Dendra2 photoswitching through the Mammary Imaging Window," Journal of Visualized Experiments, no. 28, 2009.

[42] W. V. Ingman, J. Wyckoff, V. Gouon-Evans, J. Condeelis, and J. W. Pollard, "Macrophages promote collagen fibrillogenesis around terminal end buds of the developing mammary gland," Developmental Dynamics, vol. 235, no. 12, pp. 3222-3229, 2006.

[43] C. A. Carter and L. S. Ehrlich, "Cell biology of HIV-1 infection of macrophages," Annual Review of Microbiology, vol. 62, pp. 425-443, 2008.

[44] A. Mantovani, A. Sica, P. Allavena, C. Garlanda, and M. Locati, "Tumor-associated macrophages and the related myeloid-derived suppressor cells as a paradigm of the diversity of macrophage activation," Human Immunology, vol. 70, no. 5, pp. 325-330, 2009.

[45] M. Altfeld, L. Fadda, D. Frleta, and N. Bhardwaj, "DCs and NK cells: critical effectors in the immune response to HIV-1," Nature Reviews Immunology, vol. 11, no. 3, pp. 176-186, 2011.

[46] N. Romo, G. Magri, A. Muntasell et al., "Natural killer cell-mediated response to human cytomegalovirusinfected macrophages is modulated by their functional polarization," Journal of Leukocyte Biology, vol. 90, no. 4, pp. 717-726, 2011.

[47] M. M. Tiemessen, A. L. Jagger, H. G. Evans, M. J. C. van Herwijnen, S. John, and L. S. Taams, "CD4 ${ }^{+} \mathrm{CD} 25^{+} \mathrm{Foxp}^{+}$regulatory $\mathrm{T}$ cells induce alternative activation of human monocytes/macrophages," Proceedings of the National Academy of Sciences of the United States of America, vol. 104, no. 49, pp. 1944619451, 2007.

[48] N. D. L. Savage, T. de Boer, K. V. Walburg et al., "Human anti-inflammatory macrophages induce Foxp $^{+} \mathrm{GITR}^{+} \mathrm{CD} 25^{+}$regulatory $\mathrm{T}$ cells, which suppress via membrane-bound TGF $\beta-1$, Journal of Immunology, vol. 181, no. 3, pp. 2220-2226, 2008.

[49] J. P. Edwards, X. Zhang, K. A. Frauwirth, and D. M. Mosser, "Biochemical and functional characterization of three activated macrophage populations," Journal of Leukocyte Biology, vol. 80, no. 6, pp. 1298-1307, 2006.

[50] G. Gallina, L. Dolcetti, P. Serafini et al., "Tumors induce a subset of inflammatory monocytes with immunosuppressive activity on CD8 ${ }^{+}$T cells," The Journal of Clinical Investigation, vol. 116, no. 10, pp. $2777-$ 2790, 2006.

[51] P. Sinha, V. K. Clements, and S. Ostrand-Rosenberg, "Interleukin-13-regulated M2 macrophages in combination with myeloid suppressor cells block immune surveillance against metastasis," Cancer Research, vol. 65, no. 24, pp. 11743-11751, 2005.

[52] V. Frodermann, T. A. Chau, S. Sayedyahossein, J. M. Toth, D. E. Heinrichs, and J. Madrenas, "A modulatory interleukin-10 response to staphylococcal peptidoglycan prevents Th1/Th17 adaptive immunity to Staphylococcus aureus," Journal of Infectious Diseases, vol. 204, no. 2, pp. 253-262, 2011.

[53] S. A. Miles, S. M. Conrad, R. G. Alves, S. M. B. Jeronimo, and D. M. Mosser, "A role for IgG immune complexes during infection with the intracellular pathogen Leishmania," The Journal of Experimental Medicine, vol. 201, no. 5, pp. 747-754, 2005.

[54] P. Baetselier, B. Namangala, W. Noël, L. Brys, E. Pays, and A. Beschin, "Alternative versus classical macrophage activation during experimental African trypanosomosis," International Journal for Parasitology, vol. 31, no. 5-6, pp. 575-587, 2001.

[55] O. M. Pena, J. Pistolic, D. Raj, C. D. Fjell, and R. E. W. Hancock, "Endotoxin tolerance represents a distinctive state of alternative polarization (M2) in human mononuclear cells," Journal of Immunology, vol. 186, no. 12, pp. 7243-7254, 2011. 
[56] M. Ishii, H. Wen, C. A. S. Corsa et al., "Epigenetic regulation of the alternatively activated macrophage phenotype," Blood, vol. 114, no. 15, pp. 3244-3254, 2009.

[57] T. Satoh, O. Takeuchi, A. Vandenbon et al., "The Jmjd3-Irf4 axis regulates M2 macrophage polarization and host responses against helminth infection," Nature Immunology, vol. 11, no. 10, pp. 936-944, 2010.

[58] Z. Zhang, L. Song, K. Maurer, A. Bagashev, and K. E. Sullivan, "Monocyte polarization: the relationship of genome-wide changes in $\mathrm{H} 4$ acetylation with polarization," Genes and Immunity, vol. 12, no. 6, pp. 445-456, 2011.

[59] A. H. Klimp, E. G. E. de Vries, G. L. Scherphof, and T. Daemen, "A potential role of macrophage activation in the treatment of cancer," Critical Reviews in Oncology/Hematology, vol. 44, no. 2, pp. 143-161, 2002.

[60] A. Mantovani, B. Bottazzi, F. Colotta, S. Sozzani, and L. Ruco, "The origin and function of tumor-associated macrophages," Immunology Today, vol. 13, no. 7, pp. 265-270, 1992.

[61] A. Mantovani, T. Schioppa, C. Porta, P. Allavena, and A. Sica, "Role of tumor-associated macrophages in tumor progression and invasion," Cancer and Metastasis Reviews, vol. 25, no. 3, pp. 315-322, 2006.

[62] L. Bingle, N. J. Brown, and C. E. Lewis, "The role of tumour-associated macrophages in tumour progression: implications for new anticancer therapies," Journal of Pathology, vol. 196, no. 3, pp. 254-265, 2002.

[63] J. A. Hamilton, "Colony-stimulating factors in inflammation and autoimmunity," Nature Reviews Immunology, vol. 8, no. 7, pp. 533-544, 2008.

[64] T. Hagemann, T. Lawrence, I. McNeish et al., “'Re-educating' tumor-associated macrophages by targeting NF$\kappa \mathrm{B}$," The Journal of Experimental Medicine, vol. 205, no. 6, pp. 1261-1268, 2008.

[65] T. Hagemann, S. K. Biswas, T. Lawrence, A. Sica, and C. E. Lewis, "Regulation of macrophage function in tumors: the multifaceted role of NF- $\kappa$ B," Blood, vol. 113, no. 14, pp. 3139-3146, 2009.

[66] F. Balkwill, K. A. Charles, and A. Mantovani, "Smoldering and polarized inflammation in the initiation and promotion of malignant disease," Cancer Cell, vol. 7, no. 3, pp. 211-217, 2005.

[67] L. M. Coussens and Z. Werb, "Inflammation and cancer," Nature, vol. 420, no. 6917, pp. 860-867, 2002.

[68] V. Gouon-Evans, E. Y. Lin, and J. W. Pollard, "Requirement of macrophages and eosinophils and their cytokines/chemokines for mammary gland development," Breast Cancer Research, vol. 4, no. 4, pp. 155-164, 2002.

[69] P. D. Smith, K. Ohura, and H. Masur, "Monocyte function in the acquired immune deficiency syndrome. Defective chemotaxis," The Journal of Clinical Investigation, vol. 74, no. 6, pp. 2121-2128, 1984.

[70] G. Poli, B. Bottazzi, and R. Acero, "Monocyte function in intravenous drug abusers with lymphadenopathy syndrome and in patients with acquired immunodeficiency syndrome: selective impairment of chemotaxis," Clinical and Experimental Immunology, vol. 62, no. 1, pp. 136-142, 1985.

[71] C. M. Mastroianni, M. Lichtner, F. Mengoni et al., "Improvement in neutrophil and monocyte function during highly active antiretroviral treatment of HIV-1-infected patients," AIDS, vol. 13, no. 8, pp. 883-890, 1999.

[72] S. M. Wahl, J. B. Allen, S. Gartner et al., "HIV-1 and its envelope glycoprotein down-regulate chemotactic ligand receptors and chemotactic function of peripheral blood monocytes," Journal of Immunology, vol. 142, no. 10, pp. 3553-3559, 1989.

[73] K. Kedzierska and S. M. Crowe, "The role of monocytes and macrophages in the pathogenesis of HIV-1 infection," Current Medicinal Chemistry, vol. 9, no. 21, pp. 1893-1903, 2002.

[74] D. M. Musher, D. A. Watson, D. Nickeson, F. Gyorkey, C. Lahart, and R. D. Rossen, "The effect of HIV infection on phagocytosis and killing of Staphylococcus aureus by human pulmonary alveolar macrophages," American Journal of the Medical Sciences, vol. 299, no. 3, pp. 158-163, 1990.

[75] M. G. Pittis, F. Prada, A. Mangano et al., "Monocyte phagolysosomal fusion in children born to human immunodeficiency virus-infected mothers," Pediatric Infectious Disease Journal, vol. 16, no. 1, pp. 24-28, 1997.

[76] G. Herbein, U. Mahlknecht, F. Batliwalla et al., "Apoptosis of CD8 ${ }^{+}$T cells is mediated by macrophages through interaction of HIV gp120 with chemokine receptor CXCR4," Nature, vol. 395, no. 6698, pp. 189-194, 1998.

[77] J. N. Brown, J. J. Kohler, C. R. Coberley, J. W. Sleasman, and M. M. Goodenow, "HIV-1 activates macrophages independent of toll-like receptors," PLoS One, vol. 3, no. 12, Article ID e3664, 2008.

[78] G. Peng, T. Greenwell-Wild, S. Nares et al., "Myeloid differentiation and susceptibility to HIV-1 are linked to APOBEC3 expression," Blood, vol. 110, no. 1, pp. 393-400, 2007. 
[79] G. Berger, S. Durand, G. Fargier et al., "Apobec3a is a specific inhibitor of the early phases of hiv-1 infection in myeloid cells," PLoS Pathogens, vol. 7, no. 9, Article ID e1002221, 2011.

[80] F. A. Koning, C. Goujon, H. Bauby, and M. H. Malim, "Target cell-mediated editing of HIV-1 cDNA by APOBEC3 proteins in human macrophages," Journal of Virology, vol. 85, no. 24, pp. 13448-13452, 2011.

\section{This article should be cited as follows:}

Luca Cassetta, Edana Cassol, and Guido Poli, "Macrophage Polarization in Health and Disease," TheScientificWorldJOURNAL, vol. 11, pp. 2391-2402, 2011. 


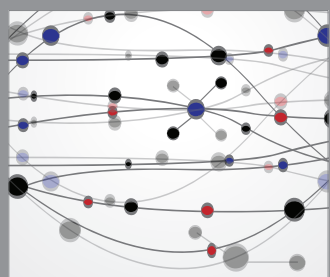

The Scientific World Journal
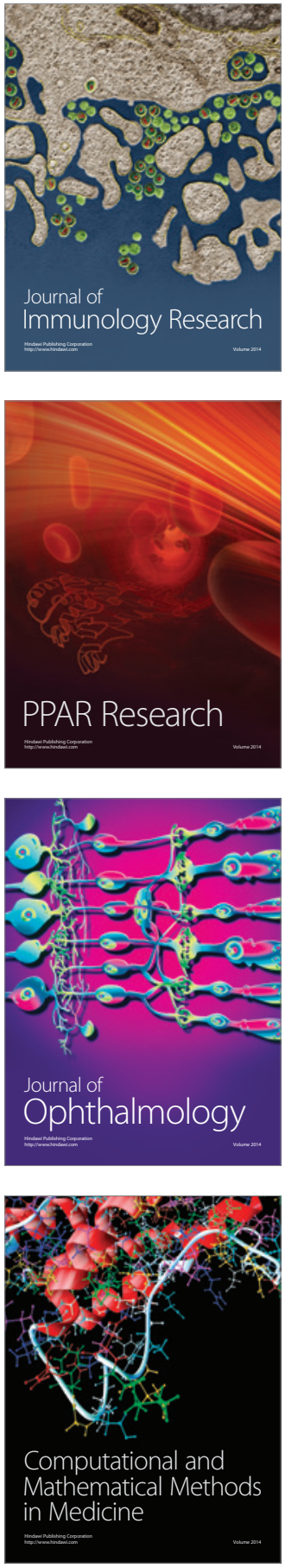

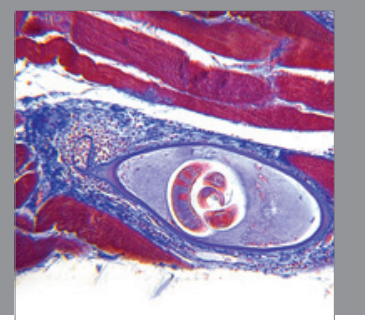

Gastroenterology

Research and Practice
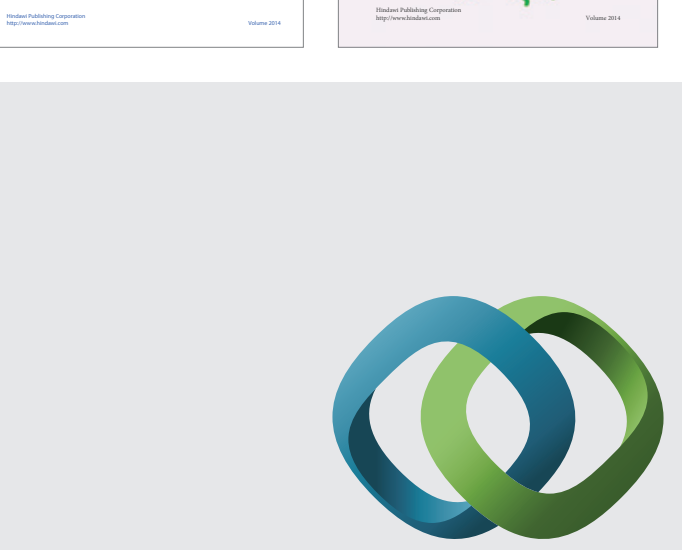

\section{Hindawi}

Submit your manuscripts at

http://www.hindawi.com
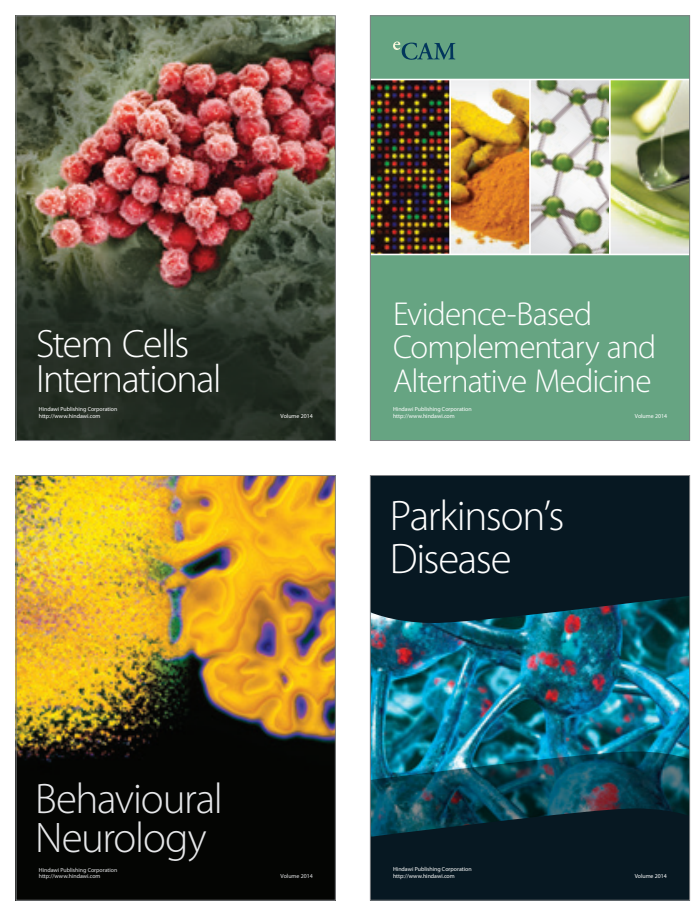

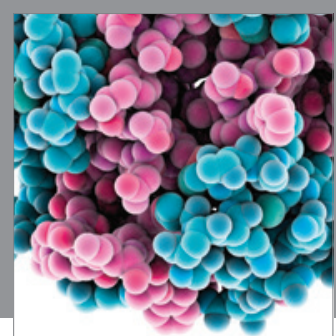

Journal of
Diabetes Research

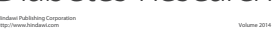

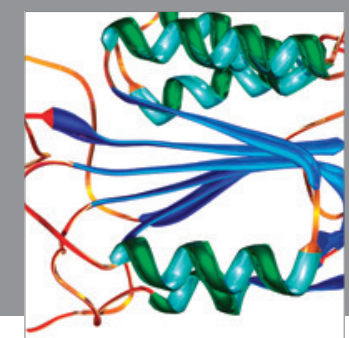

Disease Markers
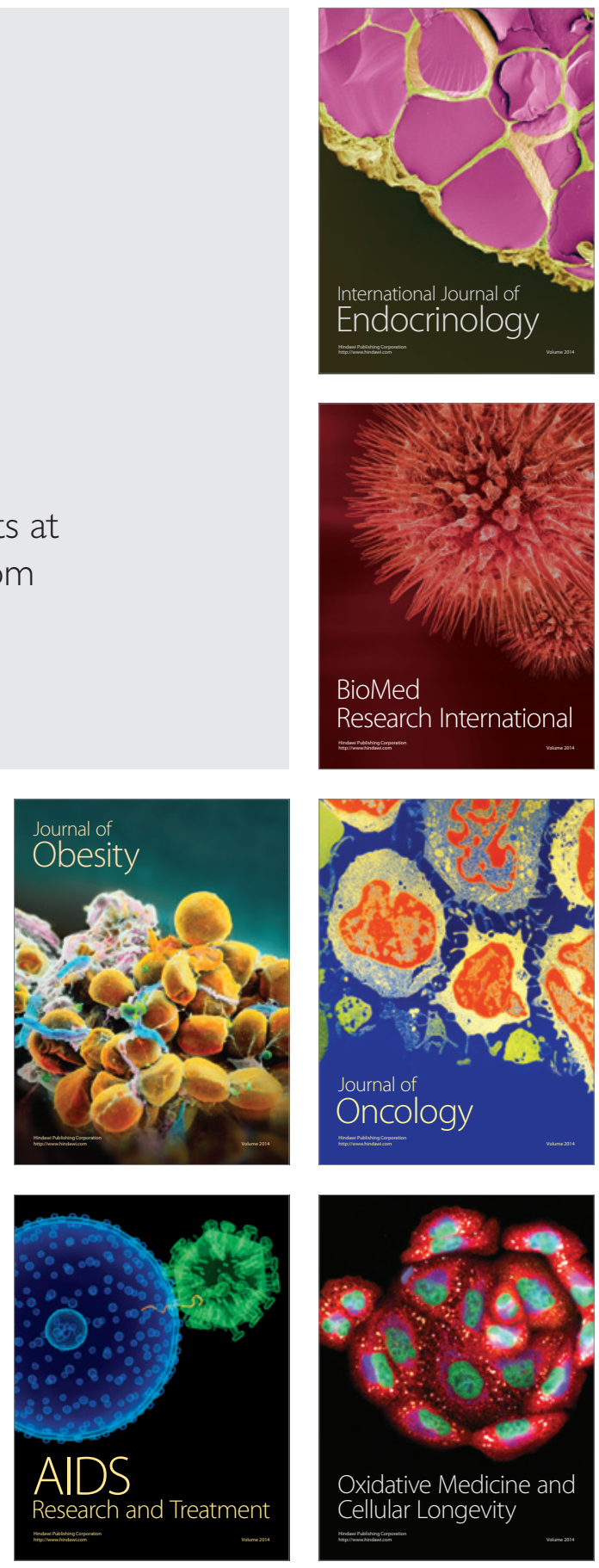